\title{
Rare case of Fanconi anemia associated with polydactly
}

\author{
Ghaida Bakoar Alahmadi ${ }^{*}$, Ibrahim Alharbi ${ }^{2}$, Imtinan kh.Alsahafi ${ }^{1}$, Bilqis Ahmed Albarakati ${ }^{1}$ and Shahad M Aldor ${ }^{1}$ \\ ${ }^{1}$ Medical Intern, Um Alqura University, Jeddah, Saudi Arabia \\ ${ }^{2}$ Consultant Pediatric Hematology Oncology, King Fahad Armed Forces Hospital, Jeddah, Saudi Arabia
}

\section{Introduction}

Fanconi anemia (FA) is a rare progressive congenital bone marrow failure syndrome. It is inherited in an autosomal recessive manner and is associated with a constellation of clinical findings. Lymphocytes and skin fibroblasts in patients with FA are characterized by hypersensitivity to DNA-cross linking agents such as Diepoxybutane (DEB) and Mitomycin C (Chromosome Breakage Test) resulting in chromosomal aberrations. Mutations in at least 20 genes have been associated with FA. Most frequent gene mutations in FA patients worldwide is FANCA which account for $60-65 \%$ of the patients.

The epidemiology and frequency of different subtypes of FA in our population are not well known. In one study of the molecular patterns of FA in ten Saudi patients showed that BRIP1 (FANCJ) was the most frequent compared to only $2 \%$ in Europeans. In Saudi Arabia, FA is one the most frequent indications for bone marrow transplantation among patients with inherited bone marrow failure syndromes [1]. Fanconi anemia can present with skeletal anomalies, bone marrow failure, and increase in the risk of malignancy. In the first decade of life, FA patients usually present with pancytopenia caused by progressive bone marrow failure [2].

\section{Case report}

A four years old Saudi girl with a history of failure to thrive, pancytopenia for six months presented with fevers for two days as well as vomiting for one day. She was admitted as a case of pancytopenia for investigations. On physical examination, her height was $92 \mathrm{~cm}(<5$ th \%) consistent with short stature. Her weight was $12.8 \mathrm{~kg}(<5$ th \%). She was also found to have bilateral microcoria as well as partial ptosis. She has hyperpigmented lesions (Café-au-lait spots). She has also been noted to have polydactly in the right hand. She has a small extra digit next to her right thumb. Other systemic examination was unremarkable.

Laboratory investigations showed Hemoglobin of $4.2 \mathrm{~g} / \mathrm{fl}$. White cell count 1.9. Platelets count of 6 . Neutrophil count was 0.4 (Absolute Neutrophil Count was 400). Renal function tests and liver enzymes were all normal. She received packed red blood transfusions and platelets transfusion. Subsequently, she was admitted on average every 2-3 weeks with pancytopenia requiring blood and platelets transfusion. She also had episodes of severe febrile neutropenia requiring admission and intravenous (IV) antibiotics.

She also had abdominal and renal ultrasound imaging which showed left ectopic kidney. Echocardiogram was done and it was unremarkable.

On 29/2/2017 bone marrow aspiration and biopsy was performed. It showed significantly hypocellular marrow with all three cell lines affected. There was no fibrosis and no blasts.
We also sent for Chromosome Breakage Tests on peripheral lymphocytes using Mitomycin C and Diepoxybutane twice and were both normal. In light of the strong clinical suspcision of Fanconi anemia, we decided to send for FA gene panel. It showed homozygous mutation in Fanconi anemia G subtype (FANC-G). Therefore, she was confirmed to have as Fanconi anemia.

\section{Discussion}

Fanconi anemia is the most common cause of inherited bone marrow failure syndromes. It is characterized by genomic instability which leads to the observed clinical manifestations. Fanconi anemia proteins are involved in cell division checkpoints and DNA repair mechanisms. They are responsible for maintaining genomic stability and cell division integrity. Defects in these genes lead to chromosomal breakage, cell cycle disturbances and increased rate of somatic mutations. Clinically, it manifests commonly as growth retardation, bone marrow failure causing pancytopenia, increased risk of malignancy, skin pigmentation, and skeletal malformations. Less commonly, it may affect eyes, gastrointestinal tract, genitourinary tract, heart, oral cavity, and central nervous system. Up to twenty FA genes have been identified so far. The more common genes are FANCA, FANCB, FANC, FANCD1 (BRCA2), FANCD2, FANCE, FANCF, FANCG, FANCI, and FANCJ (BRIP1). The less common FA genes include; FANCL, FANCM, FANCN (PALB2), FANCO (RAD51C), FANCP (SLX4), FANCQ (ERCC4), FANCR (RAD51), FANCT (UBE2T), FANCU (XRCC2), and FANCV(MAD2L2) [3,4].

FNAC-G has been localized to the short arm of chromosome p923. It was cloned by Liu et al in 1997. It was also observed that cells from patients with FANC-G show resistance to Mitomycin C [5]. Later, Van der Heijden et al. in 2003 showed that both somatic and inherited FANC-G mutations are associated with young-onset pancreatic cancer [6].

The age of onset of hematologic abnormalities may vary with the mean age reported around 8 years. An early diagnosis of FA is important as an initial step to assess the need for Hematopoietic stem cell transplant which is ideally performed before the need for more frequent regular blood transfusions [7]. In our case the patient was discovered early at 4 years of age which was an advantage to initiate treatment. She presented with classical signs of a fanconi anemia; short stature, suboptimal weight, and pancytopenia. She also has bilateral

Correspondence to: Ghaida Bakoar Alahmadi, Medical Intern, Um Alqura University, Jeddah, Saudi Arabia, Tel: 00966560290851; E-mail: alahmadighaidaa@gmail.com

Received: October 29, 2017; Accepted: November 25, 2017; Published: November 28, 2017 
microcoria with partial ptosis and polydactyly in the right hand. Also, she has hypoplasia of the thump of the other hand. After analyzing the data of 700 patients with FA in the literature, almost 300 patients had thumb anomalies such as an absent thump or hypoplastic thumb, floating or bifid thumb, polydactly, as well as triphalangeal thumbs. The association of thumb abnormalities and FA has been well established $[8,9]$.

After BM aspiration was done it showed decrease in all cell lines. Then gene studies showed FANCG gene mutation in our patient. This gene mutation affects about $10 \%$ of all FA cases compared to the more detected mutation which is FANCA. FANCA is the most frequent mutation in patients with fanconi anemia accounting for about $70 \%$ of FA cases [10].

Patients with FANC-G gene mutation had more severe pancytopenia and a higher incidence of malignancy. Our case started to have pancytopenia at the age of 6 months which required frequent monitoring and early intervention to avoid the poor outcome [11].

\section{Conclusions}

In conclusion, this is a rare presentation for a FA patient having polyductaly and thump hypoplasia, with a non-common subtype of gene mutation FANCG was detected early, such cases must be reported from Saudi Arabia, to avoid the poor hematologic outcome and to identify the etiology of this association between specific subtypes of gene mutations and their outcome, further genetic mutation analysis for FA is needed.

\section{References}

1. Ghazwani Y, AlBalwi M, Al-Abdulkareem I, Al-Dress M, Alharbi T, et al. (2016) Clinical characteristics, and genetic subtypes of Fanconi anemia in Saudi patients. Cancer Genet 209: 171-176. [Crossref]

2. Alter BP, Kupfer G. Fanconi anemia (2013) In: Pagon RA, Adam MP, Ardinger HH, et al. (Eds.), Gene Reviews. Seattle, WA: University of Washington.

3. Aymun U, Iram S, Aftab I, Khaliq S, Nadir A (2017) Screening for mutations in two exons of FANCG gene in Pakistani population. Biomed Pap Med Fac Univ Palacky Olomouc Czech Repub 161: 158-163. [Crossref]

4. Bluteau D, Masliah-Planchon J, Clairmont C, Rousseau A, Ceccaldi R, et al. (2016) Biallelic inactivation of REV7 is associated with Fanconi anemia. J Clin Invest 126: 3580-3584. [Crossref]

5. Liu N, Lamerdin JE, Tucker JD, Zhou ZQ, Walter CA, et al. (1997) The human XRCC9 gene corrects chromosomal instability and mutagen sensitivities in CHO UV40 cells. Proc Natl Acad Sci USA 94: 9232-9237. [Crossref]

6. van der Heijden MS, Yeo CJ, Hruban RH, Kern SE (2003) Kern, Fanconi Anemia Gene Mutations in Young-onset Pancreatic Cancer. Cancer Res 63: 2585-2588. [Crossref]

7. Afshar A (2016) Fanconi Anemia Concurrent with an Unusual Thumb Polydactyly: A Case Report. Arch Bone Jt Surg 4: 185-187. [Crossref]

8. Alter BP (1992) Arm anomalies and bone marrow failure may go hand in hand. $J$ Hand Surg Am 17: 566-571. [Crossref]

9. Wilks DJ, Kay SP, Bourke G (2012) Fanconi's anaemia and unilateral thumb polydactyly--don't miss it. J Plast Reconstr Aesthet Surg 65: 1083-1086. [Crossref]

10. Solomon PJ, Margaret P, Rajendran R, Ramalingam R, Menezes GA, et al. (2015) A case report and literature review of Fanconi Anemia (FA) diagnosed by genetic testing. Ital J Pediatr 41: 38. [Crossref]

11. Faivre L, Guardiola P, Lewis C, Dokal I, Ebell W, et al. (2000) Association of complementation group and mutation type with clinical outcome in Fanconi anemia. Blood 96: 4064-4070. [Crossref]

Copyright: (C2017 Alahmadi GB. This is an open-access article distributed under the terms of the Creative Commons Attribution License, which permits unrestricted use, distribution, and reproduction in any medium, provided the original author and source are credited. 